\title{
B
} Lawrence Berkeley Laboratory UNIVERSITY OF CALIFORNIA

Physics Division

Presented at the Internatonal Symposium on

Suangeness in Hadrome Matter.

Bad Honnef. West Gormany. Inne 1-5. 148?

Production of Strange Particles

in Hadronization Processes

H. Hoinann

fugusi 1987

REPFODUCED FROM

BEST AVAILAELE COPY

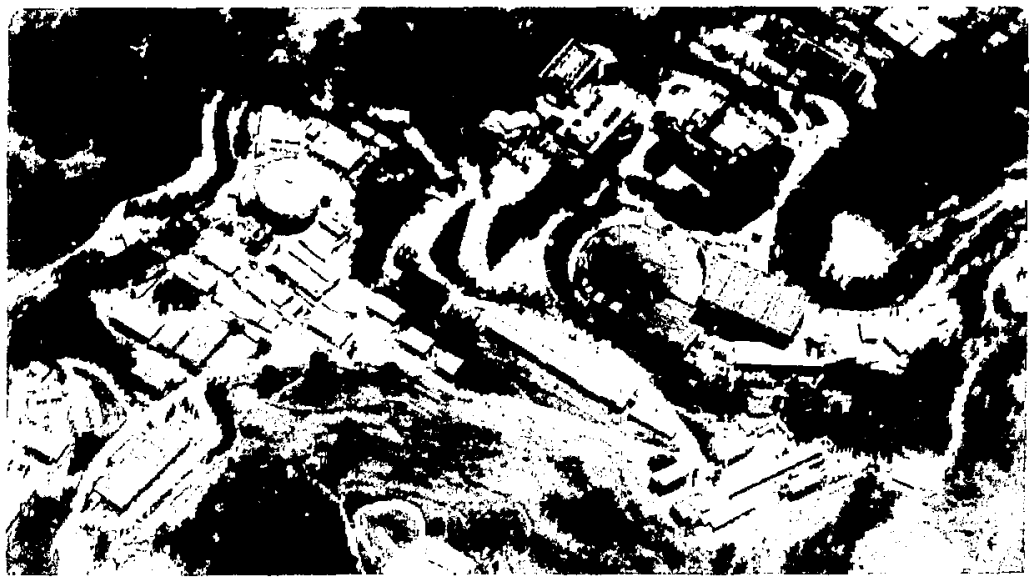


This report was prepared as an account of work sponsored by an agency of the Unitcd States Government. Neither the United States Government nor any agency thereor, nor any of their employees, makes any wisranly, express or implied, or assumes any legal liability or responsibility for the accuracy, compleleness, or usefulness of any information, apparatus, product, or process disclosed, or represents that its use would not infringe privately owned rights. Reference herein to any specific commercial product, process, or service by trade name, trademark. manufacturer, or otherwise dows not necessarily constitute or imply its endorsemen1, recommendation. or Fuvoring by the United States Government or any agency thereof. The views and opinions of authors expressed herein do not necessarily state or reflect those of the United States Government or any ageney thereor.

\title{
Production of Strange Particles in Hadronization Processes
}

\author{
W. Hofmann
}

LBL- -23921

DE88 000461

\author{
Latvrence Berkeley Laboratory \\ University of Califomia \\ Berkeley, CA 94720
}

August 1987

Invited Talk at the

Intemational Symposium on Strangeness in Hadronic Matter

1-5 June 1987, Bad Honnef, FRG 


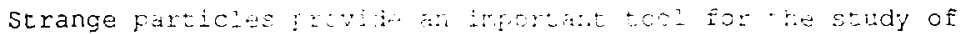

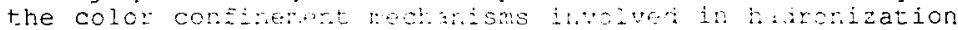

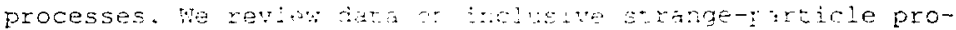

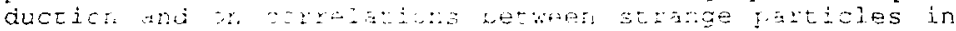

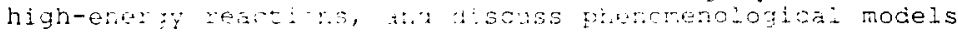

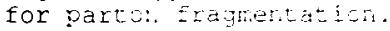

1. INTRODUCIICN.

Purpose of this vater ij to review data and models on the production af arang perticias in high-energy reactions such as

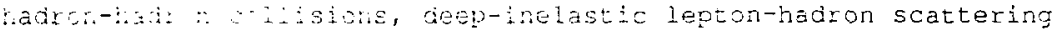

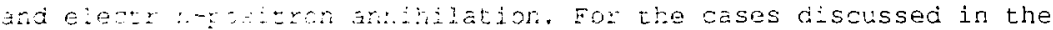

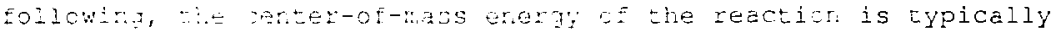
much taryt: and sanage-radren masses, and the production rates of strange fa: actos should reflect the dyamics of the strong interaction rather than piase space constraints.

Since so far most talks at this conference have dealt with strangeness in auciear physics, I would like to review briezly the phenomenology of high-energy reactions and point olit some of the reasons why prcidczion rates and production characteristics of strange particies are of considerable interest. Probably the most typical feature of high-energy reactions is the formation of jets, i.e. the emission of particles in well-collimated bundes. In eloctron-positron annin: laticn into hadrons in the EEP/PETRA energy range, For ełample, cne fypically finds two jets recoiling agains: each other, as exemplified by the "typical" event shom in Eig. 1. OE course, the cwo jets reflect the production of a irinary guarkantiquark pair by a virtual photon; as the tro quarks receie from each other, a confining color force field compled to tineir su(3) color charges prevents chem from escaping as asymacta particles and instead results in the production of jets of hadrors collimated around the parton directions.

The mechanism of color confinement is one of the bi cker guestions ir particle paysics and has attracted consicieraye ittertion, so far without complete success. Tne study of haciorization processes in general, and of strange-particle production in perticuiar 

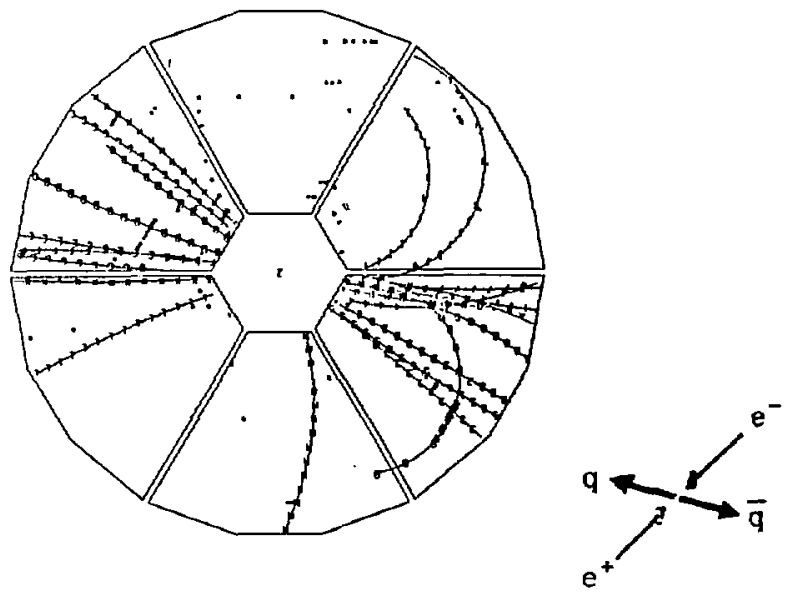

EIGURE 1

A typical 2-jet event in $\mathrm{e}^{+} \mathrm{e}^{-}$annihilation at $\sqrt{s}_{\mathrm{s}}=29 \mathrm{GeV}$, viewed along the $e^{+} e^{-}$collision axis.

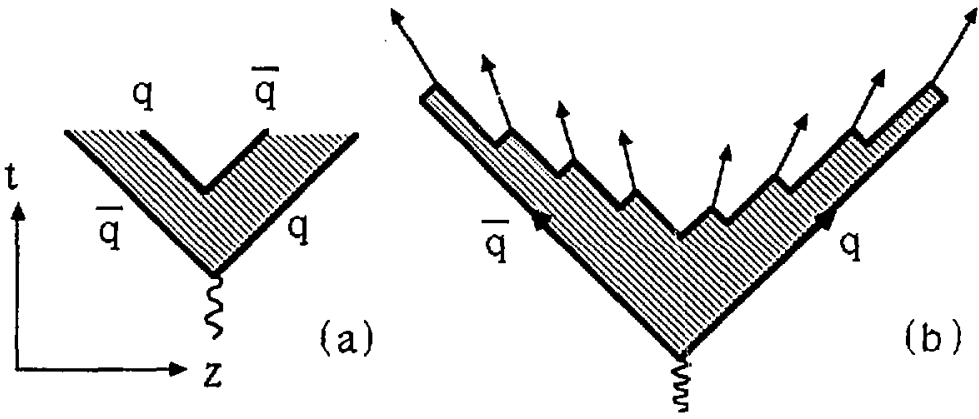

EIGURE 2

Particle production in the constant color force field between quark and antiquark: (a) production of a new quark-antiquark pair; (b) a complete annihilation event. Since the creation of a new guark pair will on average occur after a constant proper time, particle production points are scattered about a space-time hyperbola. 
provides one of the handles towards a deeper understanding uf the strong forces responsible for confinement:

- It is presently believed that the confining potential between a quark and an antiquark rises linearly with distance, i.e. the force field can be pictured as a basically one-dimensional field, a "color flux Fube" or "string", often compared to vortez lines in a superconductor. In such a model1-5, confinement arises since once the primary quarks are separated by a certain distance (of the order of one Eermil, it is energetically favorable to form a new guak-antiuark pair which screens the color field (Eig. 2(a)). At hign energies, the process is repeated several times until the mass ot each quark-antiquark system joined by a string piece is consisent with typical meson masses (Fig. 2 (b)). In such a process, the rate of strange quark production as compared to production rates for light up or down quarks depends on the enes $\because$ density in the color field $2,4-7$. The ratio of strange to up or down quark rates provides therefore a measurement of this energ; densit $z$, the "string constant", or in other words of the typical scales of space, time and momentum transfer involved in confiremert processes.

- The strangeness quancum number can also be used as a tracer to explore reaction dynamics. In a typical high-energy reaction, 10 or more pions are produced in the confinement process, but typically only one of two pairs of strange particles. The position in phase-space of a strange hadron with respect to its associated anti-strange partner provides clues ta the momentum transfers in the confinement process (Fig. 3). In this context, it is also helpful that thore are few resonances decaying into pairs of strange particles, whereas the observed correlations between pions are largely caused by resonance decays.

- In general strange particies, because of their larger masses, make a more reliable probe of the fragmentation process. Most pions stem from resonance decays. Since in a typical pion producirg decay such as $\rho \rightarrow \pi \pi$ the $Q-v a l u e$ of the decay is larger than the pion mass, the momentum of the final-state pion is a very poor 


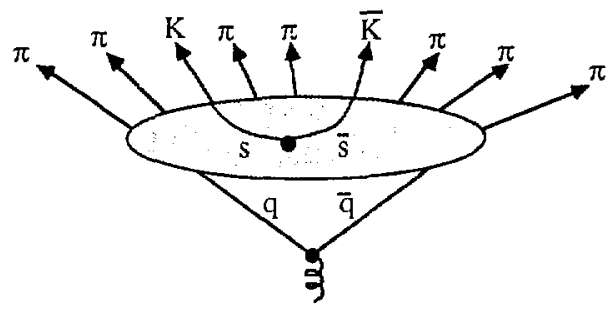

EIGURE 3

The strangeness quantum number can be used to label pairs of hadrons containing pair-produced quarks. The four-momentum difference of those hadrons measures the typical scale of momentum transfer in the confinement process.

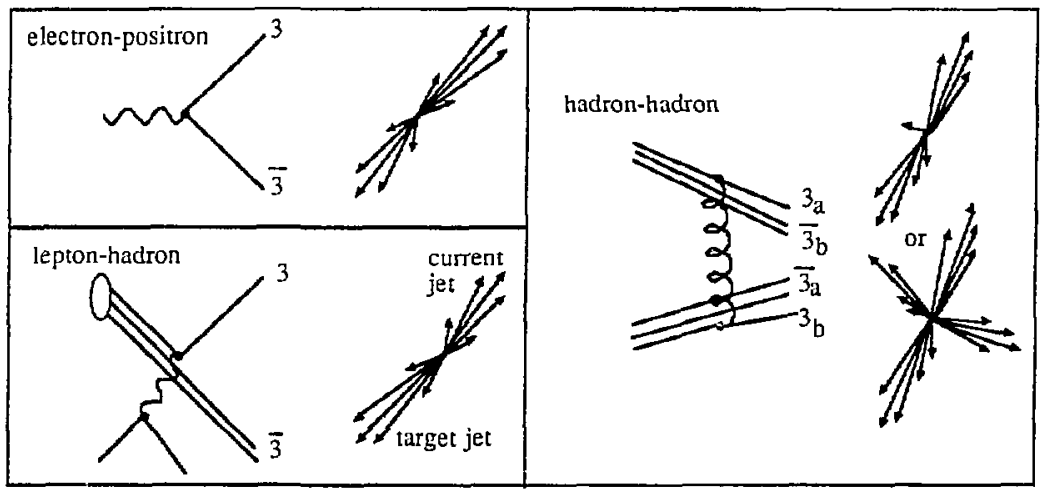

FIGURE 4

Underlying partonic processes in high energy reactions and the resulting jet structures of the events: (a) $e^{+} e^{-}$annihilation into two jets; (b) lepton-rucleon scattering with current jet and target jet; (c) hadron-hadron collisions.For large momenta of the exchanged gluon, a four-jet structure is visible, corresponding to the four color triplets created in the process. For small momenta, pairs of jets coalesce. (Another possible process is of course the scattering off gluons.) 
estimator of its parent's momentum. This situation improves as we go to strange mesons. (E.g. consider $K^{*} \rightarrow K \pi$. )

- Whereas meson production appears very natural in most models of the confinement mechanism, the mechanisms responsible for baryon production are by no means obvious 8,9 . Baryon production rates, with the number of strange quarks in the baryon between 0 and 3 , provide an excellent testing ground for models and allow systematic studies.

- Finally, strange baryons enable us to study polarization effects in the hadronization process. In particular, the $\Lambda$ baryon polarization is easy to measure and provides a direct measurement of the polarization of the s-quark. String models, for example, do predict a significant polarization of secondary quarks produced in the color fieldio.

It is believed that the separation of color charges, followed by a confinement process resulting in a two- or multijet structure is one of the fundamental mechanisms of particle production in highenergy reactionsll. Fig. 4 indicates the underlying processes in various reactions: $e^{+} e^{-}$annihilation into quark and antiquark, resulting usually in a two-jet event; deep-inelastic lepton nucleon scattering where a guark is scattered out of a nucleon and where the remaining diguark acts as a color-antitriplet, resulting in a current jet and a target jet; finally hadron-hadron collisions, where the underlying mechanism is not really known, but a reasonable (somewhat simplified) guess is that a gluon is exchanged, giving rise to two triplet-antitriplet systems and hence four jets. For low momentum transfers, pairs of jet overlap and we are left with a beam and a target jet. For bigh momenta of the exchanged gluon, a four jet structure is predicted and indeed observed.

The kinematics of particles within jets are typically described using a transverse variable, such as the transverse momentum with respect to the jet axis, and a longitudinal variable such as the rapidity $y$ :

$$
Y=\frac{1}{2} \log \left(\frac{E+p \|}{E-p \|}\right) .
$$


Rapidity is basically the relativistic equivalent of the velocity $\beta$ in non-relativistic systems: rapidity differences are invariant under longitudinal boosts, and particles from the decay of a common parent have the same average rapidity and typically a small rapidity difference or $O(1)$. Furthermore, a small rapidity range dy corresponds to the volume element dp/E of Lorentz invariant longitudinal phase space. It is therefore not surprising that particles in jets exhibit a basically flat distribution in rapidity, falling off near the kinematic limits. For example, in $\mathrm{e}^{+} \mathrm{e}^{-}$annihilation at PEP or PETRA energies, the kinematically allowed range for pions is \pm 5 units in rapidity; one finds 12 a central plateau of \pm 2 units, followed by "fragmentation regions" at both ends (Fig. 5). The fragmentation regions are populated mainly by particles containing tne initial parton(s), i.e. either the primary quark or the antiquark in the case of $e^{+} e^{-}$annihilation. The central region contains mainly particles made of newly formed quarks. A very clear example for the distinction between central region and fragmentation region is given by pp interactionsl3 (Eig. 6): in the central region of about \pm 2 units in rapidity, the average charge of hadrons is close to zero, indicating that they do not contain valence quarks of the incoming protons. At higher rapidity, we find the remnants of the beam protons, resulting in a positive average charge of the produced particles. Here, we will concentrate on the central region. with the exception of Iepton-nucleon scattering, the fragmentation regions are of limited use for the study of strangeness production: in $e^{+} e^{-}$annihilation, strange particle production in these regions is dominated by decay products of charmed and bottom hadrons, and in hadronic reactions the fragmentation regions usually contain complicated multi-parton systems.

In the main body of this paper, I will address the following topics:

- Strangeness suppression in hadronization processes and its phenomenological interpretation

- Correlations between strange particles and study of particle production mechanisms

- Froduction of strange baryons. 


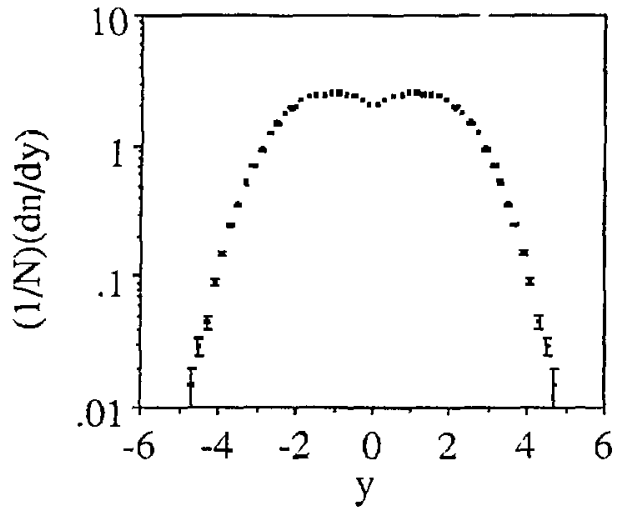

FIGURE 5

Rapidity distribution of charged hadrons in $e^{+} e^{-}$annihilation at $\sqrt{s}_{5}$ $=34 \mathrm{GeV}^{12}$. The dif at $y \approx 0$ is largely due to a bias incroduced by the algorithm used to define the jet axis (thrust axis).
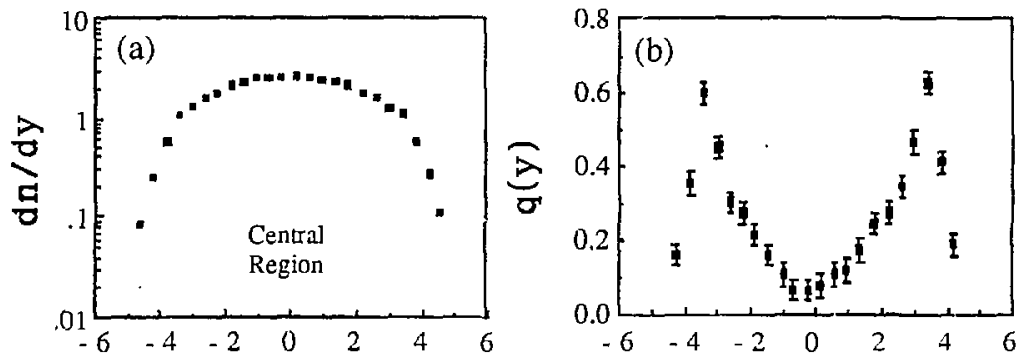

Rapidity $y$

FEIGURE 6

(a) Rapidity distribution of charged hadrons in proton-proton collisions at $V_{s}=52 \mathrm{GeV}^{13}$. (b) Net charge density, i.e. number of positive hadrons minus number of negative hadrons per urit of rapidity. 


\section{STRANGENESS SUPRRFSSION}

One of the first observations concerning the production of strange particles in hadronization processes was a breaking of Su(3) symmetry, as indicated by a reduction in the yields of strange hadrons as compared to non-strange particles. Figs. 7 (a) and (b) show the inclusive production cross settions for pions and for charged and neutral kaons (a) and for pions, protons, lambdas and $x i$ 's (b), for $\mathrm{e}^{+} \mathrm{e}^{-}$annihilation at $34 \mathrm{GeV}$ center-of-mass energy as a function of $x=2 E_{\text {hadron }} / V_{s} 14$. Both for mesons and baryons it is obvious that production cross sections decrease with increasing number of strange quarks in a hadron. Phenomenologically, this behavior can be accounted for by introduction of a suppression factur $\lambda$ for the production probability $\mathrm{P}(\mathrm{s} \overline{\mathrm{s}})$ of strange quark-antiquark pairs in the color field, as compared to light (up or down) pairs:

$$
\lambda=\frac{P(s \bar{s})}{P(u \bar{u})}=\frac{P(s \bar{s})}{P(d \bar{d})}
$$

(As we shall see later, the energy density in the color field is too small to produce $c \bar{c}$ or $b \bar{b}$ pairs at any appreciable rate.) Assuming that meson production can indeed be described as a two-step process - quark-antiquark pair production followed by a flavor-independent recombination into mesons - and neglecting leadingparticle effects, we can express the rate of primary mesons with open strangeness in terms of $\lambda$ :

$$
\frac{P \text { (strange meson) }}{P \text { (non-strange meson) }}=\frac{4 \lambda}{4+\lambda^{2}}
$$

Note, however, that Eqr. (2) holds only for primary particles; the stable hadrons observed in the detector result largely from resonance decays which produce many new pions, but usually don't change the number of strange hadrons. Correspondingly, the ratio of final kaons to pions differs significantly from the prediction of Eqn. (2), and depends on assumptions concerning the primary hadrons, such as the ratio of vector mesons to pseudoscalar mesons. This ract complicates the experimental determination of $\lambda$ tremen dously. One now has to solve the matrix equation 

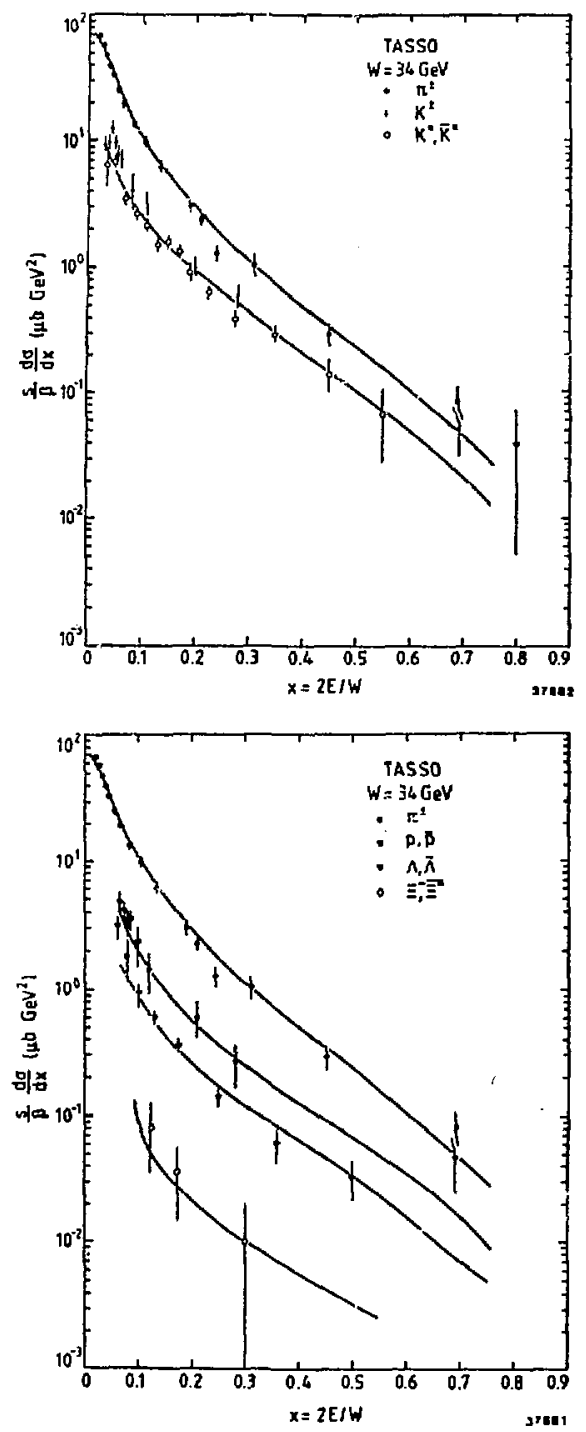

\section{EIGURE 7}

(a) Scaled cross section $(s / \beta)(d \sigma / d x)$ for production of charged pions and kaons and of neutral kaors in $e^{+} e^{-}$ annihilation at $\sqrt{s}=$ $34 \mathrm{GeV}$, as a function of $x=2 E / \sqrt{5}^{14}$. (b) Scaled cr.ss section for production of pions, protons, iambdas and cascade particles. The curves show pradictions of $t h e \quad-I$ u n d fragmentation model. with parameters oftimized for best fit to the data. 


$$
\left(\begin{array}{c}
\text { observed strange rate } \\
\text { observed non-str. }
\end{array}\right)=\left(\begin{array}{cc}
\approx 1 & \approx 0 \\
\alpha & \beta
\end{array}\right)\left(\begin{array}{c}
\text { prim. } \\
\text { prim. non-strange rate }
\end{array}\right)
$$

the matrix element $\alpha$ gives the average number of stable non-strange hadrons generated in the decay chain of a strange primary hadron, and $\beta$ gives the number from non-strange primary hadrons. Unfortunately, the nature and composition of the primary hadrons are generally not well known, and one has to rely on assumptions. Often, the quark-model meson and baryon ground states are used to represent primary particles, i.e. pseudoscalar and vector mesons and octet and decuplet baryons are included. For the production rates SU(6) symmetry is assumed, broken by the additional strangeness suppression $\lambda$ (and sometimes by additional spin- and flavor-dependent factors), and usually supplenented by a model for baryon production. The range of models is enormous - at one end the simple one-parameter $(\lambda)$ statistical modell5 (which tends to overestimate vector meson rates, indicating the need for a spin-dependent SU(6) breaking); at the other end the Iund Monte Carlo model 5 with its about a dozen parameters related to the composition of primaries. Some authors include tensor mesons among primaries; nobody so far has consistently included $\mathrm{J}^{P C}=0^{++}, 1^{++}$and $1^{+-}$mesons, given that those multiplets are not even unambiguously established. Some recent models16-19 of parton fragnentation postulate "clusters" (i.e. highly excited meson states in the $1-3 \mathrm{GeV}$ mass region, somewhat along the lines of Hagedorn's statistical bootstrap 20 ) as the primary objects produced in the process of color confinement and yield quite different values of $\lambda$.

Even once the definition of a primary particle is settled, there are still many choices. One can e.g. determine $\lambda$ by twiddling the corresponding parameter(s) in a Monte Carlo event generator or an analytical model, until agreement with the data is reached, or ont can use the model to isolate channels or kinematic regions where the relation between measured rates and $\lambda$ is very direct and where corrections are believed to be small. For example, one should not have to worry abcut resonance decay products near the phase space limit $E_{\text {hadron }} \rightarrow \sqrt{\mathrm{s}} / 2$. In the analysis of data at lower cms ener- 
gies, one has to decide whether the reduced phase space for strange particles should be included in the definitjon of $\lambda$ or not. other uricertainties concern the data used - one gan use production cross sections in the central region, inclusivefcross sections as a function of momentum, or just total multiplicities, and one can rely on some selected particle ratios (e.g. $K / \pi$ ) or instead aim for a global description of all observed cross sections.

Ideally, of course, all methods should yield the same $\lambda$; in reality, however, no model describes the data perfectly, and the answers do vary by \pm 20 to $\pm 30 \%$, depending on the analysis technique. Let me substantiate this statement with a few examples: Fig. 8 shows the predicted $\mathrm{K}^{ \pm} / \pi^{ \pm}$ratio as a function of $\lambda$, for models based on SU(6) symmetry with an additional vector meson suprression factor. Baryon production follows the Iund scheme 5 and contains additional parameters. Curves shown are for vector/pseudoscalar = $0,1: 1,3: 1$ and 1 . The $3: 1$ case is essentially equivalent to the one-parameter additive quark model15; $1: 1$ is favored by data on $\rho$ and $K^{*}$ cross sections. We see that the ratio $(\mathrm{d} \sigma / \mathrm{iy})_{K^{+}} /(\mathrm{d} \sigma / \mathrm{dy})_{\pi^{ \pm}}=$ $(9.9 \pm 1.0) \div 34$ observed in the central rapidity region of high-energy $e^{+} e^{-}$annihilation corresponds to $\lambda \approx 0.2-0.3$, depending on the assumption concerning the vector/scalar ratio.

Moreover, analytical calculations of particle rates often differ significantly from the results obtained by a full Monte-Carlo implementation. Most Iragmentation models predict that in the fragmentation region of a $d$ quark (e.g. from vp scattering) $\mathrm{K}^{0} / \pi^{-}=\lambda$ for large $\therefore=2 \mathrm{E} / \sqrt{\mathrm{s}}$. A Monte Carlo simulation however demonstrates that corrections are non-negligible, in particular et the low $\sqrt{\mathrm{s}}$ values typical for neutrino experiments (Eig. 9 (a)).

An apparently less model-dependent technique was used in a summary by Wroblewski21: he simply counts the number of strange quarks observed in hadrons (assuming that no new strange quarks are created by resonance decays), and, based on measured resonance rates and known decay multiplicities, estimates analytically the number of primary particles. The strange-hadron count includes charged and neutral kaons, lambdas, and eta's, the latter weighted by 0.5, to account for the $\approx 50 \%$ s $\bar{s}$-component in their wave function. Small 


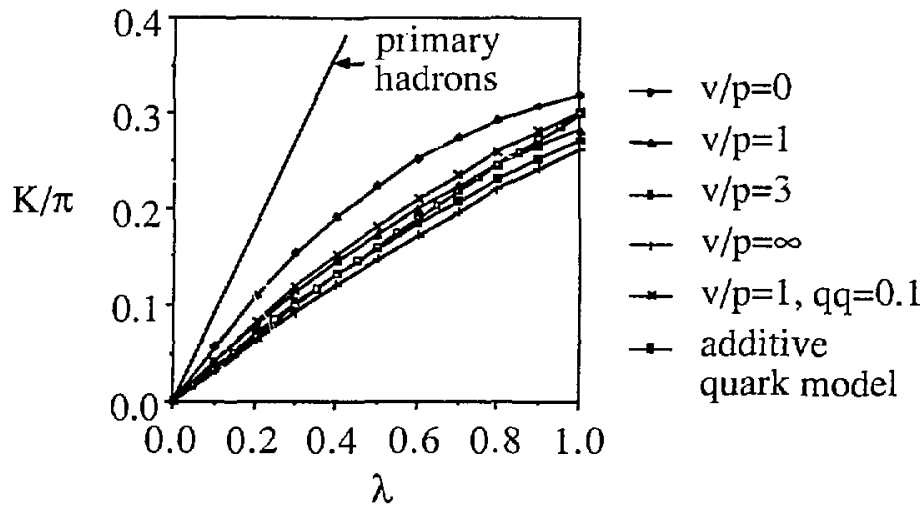

FIGURE 8

Ratio of charged kaons to pions as a function of the strangenesssuppression $\lambda$, for different ratios $v / p$ of vector mesons to pseudoscalar mesons among the primary hadrons. The $v / p=1$ predictions also shown for the assumption of occasional baryon production among primaries, implemented following the Lund diquark $s$ sheme 5 and using $\mathrm{gq} / \mathrm{q}=0.1$. The results are deriver analytically taking into account the known decay chains. The line indicates the ratio of strange to non-strange primary particles.

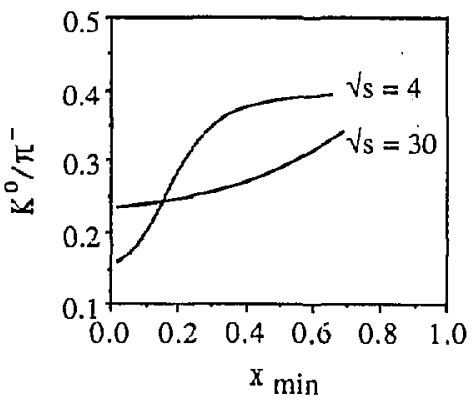

\section{FIGURE 9 (a)}

Prediction of the Lund Monte-Carlo generator 22 for the ratio of inclusive $K^{0}$ and $\pi^{-}$cross sections for $x>x_{\min }$ in the d-quark jets, for energies $w$ of the hadronic system of $4 \mathrm{GeV}$ and $30 \mathrm{GeV}$. The simulation used $\lambda=0,30$. In the limit $x_{\text {min }} \rightarrow 1$, the ratio should approach $\lambda$. 
corrections are applied for the strange baryons which have neither kaon nor lanioda in their decay chains. When applied to ū events from a Monte-Carla generator 22 (using $\lambda=0.30$ as inpur), the technique overestimates $\lambda$ considerably (Fig. 9 (b)). The reason is simple: even though eta mesons contain strange quarks, they can be

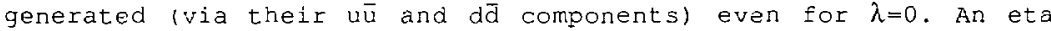
meson is thus not necessarily equivalent to 0.5 produced $5 \bar{s}$ pairs!

A Einal problem in the case of $e^{+} e^{-}$annihilation is the large strangeness production in charm and bottom decays, which at present energies account for $\approx 30 \%$ of strange-particle yields. Except for the D's, however, decay channels are not well known and the uncertainty on this correction is big. Overestimates in the kaon rate From charm resulted in significant underestimates of $\lambda$ in some early measurements.

Let us turn to measurements, and first consider strangeness production in $e^{+} e^{-}$anninilation. Fig. 10 shows a compilation 23 of the average rumber of $k^{0}$ 's per event as a function of the cms energy $\sqrt{s}$, compared to predictions of the Lund model 5 for $\lambda=0.3$. Also indicated are the contributions from primary strange quarks and from decays of charmed and bottom hadrons, which are of course energy-independent, except for thresholds, we notice that the model represents the energy dependence of the $k^{0}$ rate reasonably well using $\lambda=7.2$. Various $e^{\dagger} e^{-}$experiments $12,23-26$ have measured $\lambda$ at fired $v$ is their $\lambda$ values are in most cases obtained from fits of Lunj rode: predictions $t o k$ and $\pi$ inclusive cross sections, and are summarized in Fig. 11 (a). Here as well as in the following figrres, the error bars are of limited significance, since some experiments give just the statistical errors, whereas others include the effects of uncertainties in (some) other model parameters, whin inEluence the reasured $\lambda$. Eew analyses study the effects of yar tions of all model parameters within experimental limits, or discuss the dependerice of the measured $\lambda$ on the hadronization model. Realistic systematic erzors for most results are in the neighorhood of 20 or more.

Strange-particle production has also joen studied evtensively in lepton-nucleon scattering 27-32. There, uhe iduntity of che Erag- 

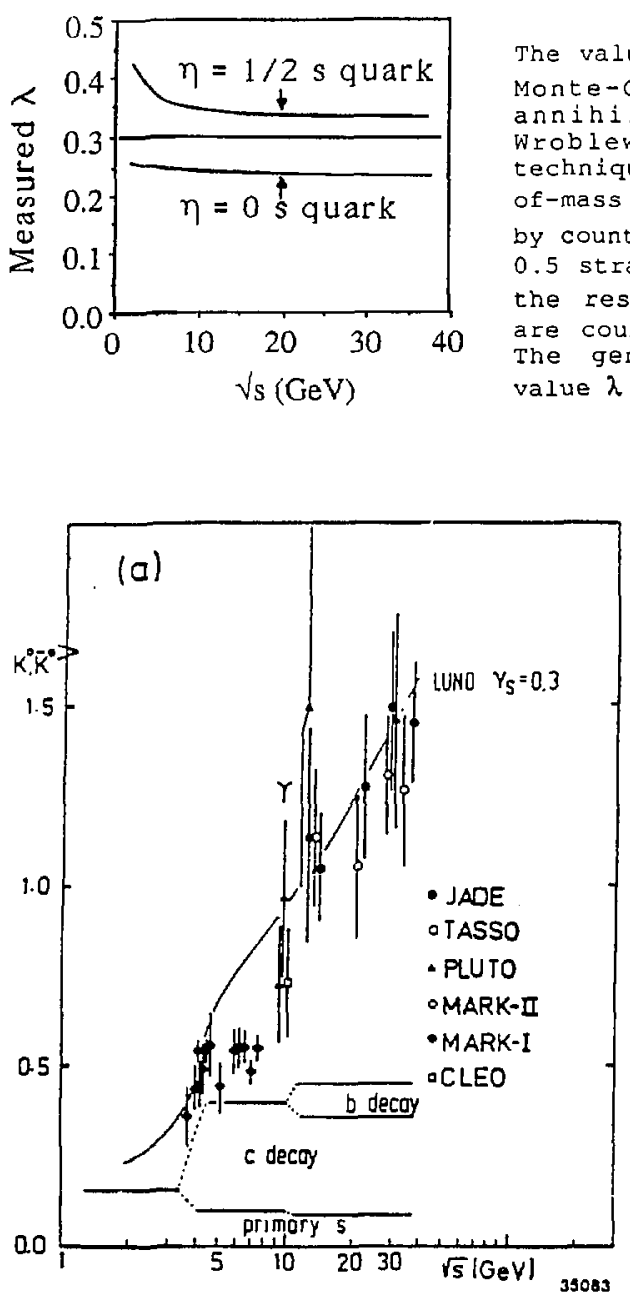

\section{FIGURE 9 (b)}

The value of $\lambda$ reconstructed from Monte-carlo generated $e^{+} e^{-}$ annihilation events using Wroblewski's hadron-counting technique, for different centerof-mass energies. $\lambda$ was calculated by counting $\eta$ mesons as containing 0.5 strange quarks. Also shown is the result obtained if $\eta$ mesons are counted as o strange quarks. The generator used a constant value $\lambda=0.3$.

\section{FIGURE 10}

Average number of neutral kaons per $\mathrm{e}^{+} \mathrm{e}^{-}$ annihilation event as a function of the center-of-mass energy $\sqrt{s} 23$. Also shown is the prediction by the Lund Monte Carlo generator for $\lambda=0.3$, and the contributions due to primary strange, charm and bottom quark pairs. 


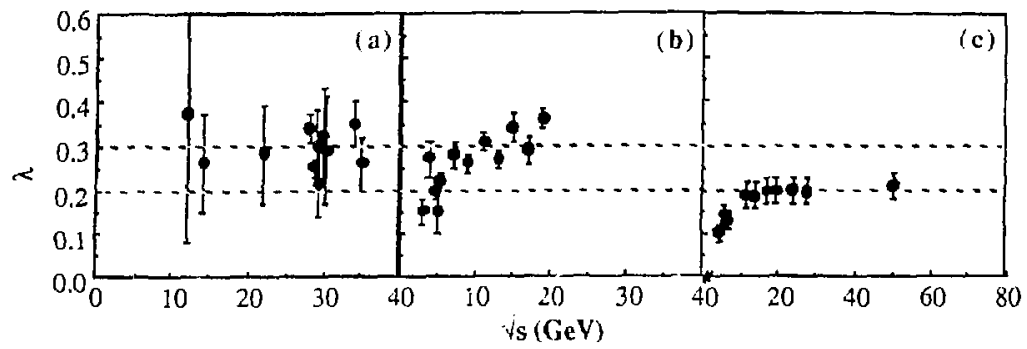

FIGURE 11

(a) Measured values of $\lambda$ as a function of center-of-mass energy, for $\mathrm{e}^{+} \mathrm{e}^{-}$annihilation events12,23-26, (b) for deep-inelastic lepton-nucleon scattering 27-32 and (c) for proton-proton reactions ${ }^{17}$. Not all errors include systematics (see text).

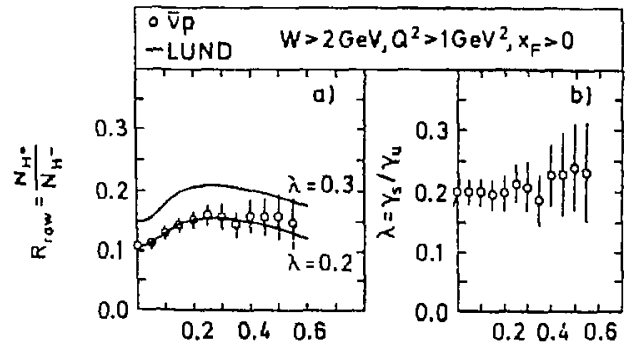

EIGURE 12

(a) The uncorrected ratio of neutral kaon rate and rate of negative hadrons as a function of $z_{m i n}$, for $v p$ (bottom) and $\bar{v}_{p}$ (top) events27. Curves show the predictions of the Lund Monte Carlo for $\lambda=0.2$ and $\lambda=$ 0.3 . (b) The values of $\lambda$ obtained by linear interpolation from the Monte-Carlo curves in (a), vs $z_{\min }$. 
menting quark is (at least approximately) known, and $\lambda$ is usually determined in the current fragmentation region. For fast hadrons, the ones most likely to contain the scattered quark plus another newly created quark, the strange/non-strange ratio provides a rather direct measurement of $\lambda$ (see also Fig 9 (a)). Fig. 12 shows data from $\mathrm{BEBC}^{27}$ : the ratio $\mathrm{R}$ of the $\mathrm{K}^{0}$ production rate to the rate of negative hadrons as a function of the minimum $z$ above which particles are included in the analysis. $z$ is defined as the ratio of hadion and scattered quark momentum; requiring high $z$ should select leading hadrons. In Fig. $12, R$ is compared with Lund model predictions for d fferent $\lambda$ and the cptimum value of $\lambda$ is derived by interpolation. Eoth the $v p$ and $\bar{v}_{z}$ data point to $\lambda \approx 0.2$; the $\lambda$ values derived for the tro data sets are consictent with each other and largely independent of the cat in $z$. Fig. 13 demonstrates further that the same $\lambda$ is obtained for samples with different Bjorken- $x$, momentum transfer $Q^{2}$, Einal-state mass $w$, charged multiplicity, or transverse momentum $\mathrm{P}_{\mathrm{T}}$ of a particle. This indicates that the Lund model does provide a consistent description of the data. As a final result, $\lambda=0.203 \pm 0.014$ (stat) \pm 0.010 (syst) is quoted; the systematic error refers to acceptance uncertainties and variations in some of the model parameters. The authors note that even at high $z$ differences between the full Lund Monte Carlo simulation and simplified analytical models are of the order $30 \%$, a fact neglected e.g. in the analysis of ref. 28, where a higher value $\lambda=0.27 \pm 0.04$ is obtained, while measured particle ratios are consistent with the $\mathrm{BEBC}$ data.

In deep-inelastic muon-proton scattering at somewhat higher average $w$, the EMC collaboration 25 finds $\lambda \approx 0.30 \pm 0.01$ (stat) \pm 0.07 (syst), using basically the same iund moclel and similar fitting techniques as for the BEBC data (Eig. 14). The $\lambda$-values obtained in lepton-nucleon scattering as a function of the invariant mass $w$ of the hadronic system are summarized in Eig. $11(\mathrm{D})$.

Einally, consider hadronic reactions. I will firet try to demonstrate in a rather model-independent way that there are no drastic differences in the strarge-particle production rates between low-pr 

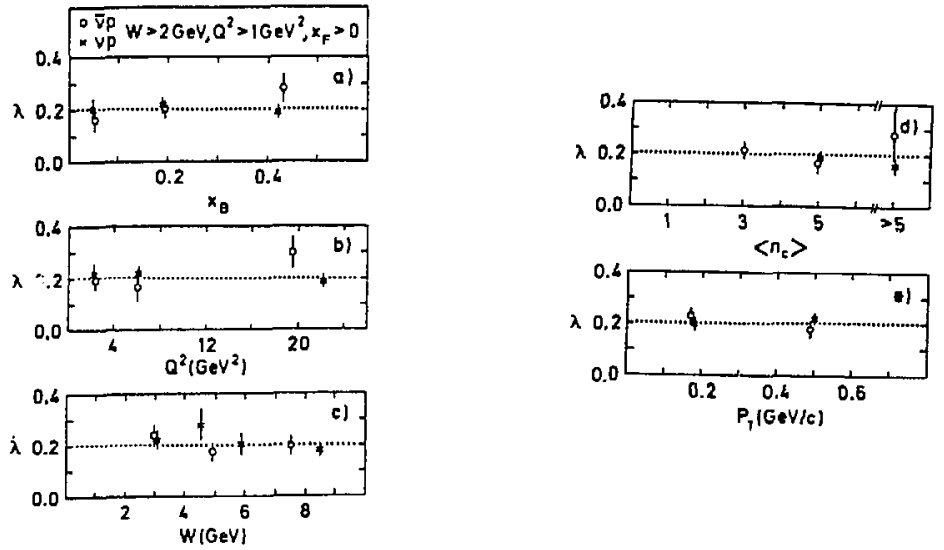

EIGURE 13

The interpolated $\lambda$ at $z_{\mathrm{min}}=0$ for $v p$ and $\bar{v}$ scattering as a function of (a) $x_{B}$, (b) momentum transfer $Q^{2}$, (c) hadronic mass $w$, (d) total charged multiplicity $\left\langle n_{C}\right\rangle$ and (e) transverse momentum $P T$ of the hadrons. The horizontal dotted Iines show $\lambda=0.20$ which is the average value determined from the combined data sample 27 .

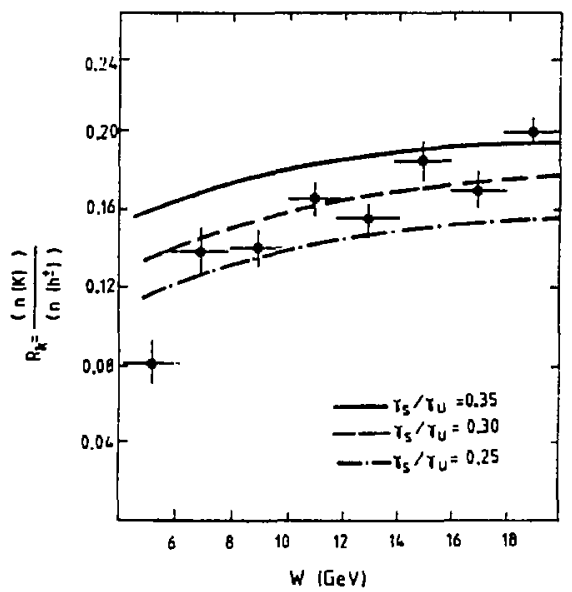

EIGURE 14

Ratio of the average total kaon multiplicity to the total charged particle multiplicity as a function of the mass $W$ of the hadronic system generated in deep-inelastic muonnucleon scattering reactions 29 . The curves show Lund model predictions for $\lambda$ values of $0.25,0.30$ and 0.35 . 
hadronic reactions and large- $q^{2}$ processes such as $e^{+} e^{-}$annihilation. We compare in Fig. 15 the $k / \pi$ ratio in pp and ppinteractions 33 and in $e^{+} e^{-}$annihilation at PEP34. In order to stay away from the highly process-specific fragmentation regions, data from the central region $y=0$ is used. While the $e^{+} e^{-}$data point ialls slightly above the pp data, it is consistent within errors, in particular given the discrepancies between different pp experiments -an indication that their systematic errors might be underestimated. One should further note that in hadronic c-ilisions about half of the incident energy is carried away by leading baryons and does not contribute to the production of new particles; particle production in pp collisions at a given $\sqrt{5 \mathrm{pp}}$ should be compared to $\mathrm{e}^{+} \mathrm{e}^{-}$annihilation at $\sqrt{\mathrm{s}} \approx \sqrt{\mathrm{spp}} / 2$. Wroblewski2l has compiled data from hadronic reactions (using the hadron-courting technique discussed above) and has derived the $\lambda$ values shown in Eig. 11(c). His method (where phase-space effects are included in $\lambda$ ) yields $\lambda \approx 0.2$, with little energy dependence once the cms energy exceeds 10 GeV (corresponding to $5 \mathrm{GeV}$ or less actually available for particle production). On the other hand, another compilation 38 derives $\lambda=0.30 \pm 0.02$ from basically the seme data, albeit using somewhat oversimplified techniques. The discrepancy between those analyses once more points to the fundamental crux ố all $\lambda$ measurements -- the strong dependence on model assumptions.

The totality of the data presented above points to a value of $\lambda$ around 0.2-0.3, without unambiguous evidence for a dependence on reaction type or cms energy, given the systematic errors of order $20-30 \%$.

The $\lambda$ value of about $0.2-0.3$ has a quite natural interpretation in the Elux-tube model $2,4-7$ discussed above. Its basic assumption a quasi one-dimensional conrinement force field - is consistent with spectroscopic data and supported by the attempts to simulate confinement on a lattice 35 . If in such a color forke field a quarkantiquark pair appears at one point along the string, energy conservation is violated by $2 \mathrm{~m}_{\mathrm{q}}$ and the system is in a short-lived, virtual state. If the quark pair has the right color to screen the initial color field, and if the quarks manage during their lifetime 


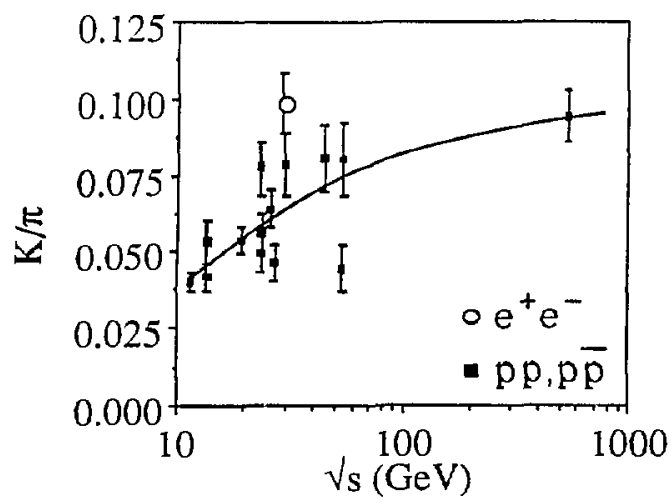

FIGLRE 15

Ratio of kaon to pion cross sections in the central rapidity region of $p p$ and $p \bar{p}$ interactions 33 , and for $e^{+} e^{-}$annihilation events 34 , as a function of the center-of-mass energy $V_{s}$.

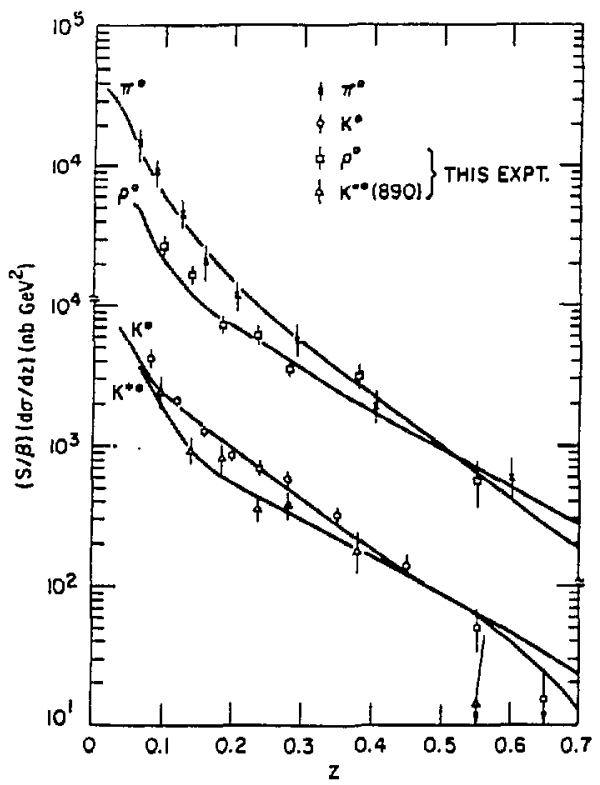

EIGURE 16

Scaled cross section for production of $\pi^{0}$, $\mathrm{K}^{0}, \rho^{0}$ and $\mathrm{K}^{* 0}(890)$ in $\mathrm{e}^{+} \mathrm{e}^{-}$annihilation at $\sqrt{s}_{\mathrm{s}}$ $=29 \mathrm{Gev}^{3} 6$. Charge conjugate states are included, where applicable. The curves show Lund model predictions for $\lambda=$ 0.34 . 
$\Delta t \approx 1 / 2 \mathrm{~m}_{\mathrm{q}}$ to separate far enough to screen an amount of field corresponding to $2 \mathrm{~m}_{\mathrm{q}}$, the quarks may end up on-shell and the string is broken. The process is basically a tunneling phenomenon, and the quark production rate depends strongly on the size of the barrier, i.e. On the mass of the newly formed quarks. One finds:

quark production rate $\approx \exp \left(-\frac{\pi \mathrm{m}_{\mathrm{g}}{ }^{2}}{\mathrm{k}}\right)$

Since the quarks are confined within a color flux tube with transverse dimensions similar to typical hadron sizes, the general expectation is that constituent quark masses of about 350 MeV for up and down quarks and of 500 to $550 \mathrm{MeV}$ for strange quarks should be used. With these masses, we can solve Eqn. (4) for the energy per unit length of the string, $k$. One finds $a$ values of $k$ around $l$ $\mathrm{GeV} / \mathrm{fm}$, well consistent with the nominal value of about $0.9 \mathrm{GeV} / \mathrm{fm}$ (corresponding to a confining force between guark and antiquark of about 10 tons) derived from the slope of Regge trajectories and other observations ${ }^{2}$. Given that value of $\kappa$, charm and bottom quark rates are surpressed by many orders of magnitude compared to jight quarks.

At this point I should come back to an earlier remark, namely the question if the strange particle suppression can really be attributed to a constant strangeness suppression factor at the quark level. In other words, do such models really give a consistent description of the data? We have already seen (Fig. 13) that within one experiment a fised value of $\lambda$ describes the data over a wide range of kinematical variables. Further checks include

- The comparison of $\lambda$ as determined from the $k / \pi$ ratios, from the ratio of strange to non-strange charmed mesons and from the ratio of strange to non-strange bottom mesons. Such a comparison tests the flavor-independence of the recombination of quarks into mesons. Unfortunately, no experimental data is available so far.

- Consistency between $k / \pi$ and $k x / p$. Within the experimental precision of about $15 \%$, data on $K, \pi, k^{*}, p^{36}$ are indeed well described by models with a constant $\lambda$ (see Fig. 16). (String models suggest $^{5}$ that the ratio $v / p$ of vector mescn production to pseu- 
doscalar meson production may be different for light and heavy quarks, complicating the interpretation of the data somewhat. However, the variation of $v / p$ between $u, d$ and $s$ quark states should be insignificant compared to present experimental errors).

- Consistency between $\phi / K^{*}$ and $K^{*} / \rho$. The suppression factor for the second strange quark should be the same as for the first one. Again models are indeed consistent with experiments 37 (Fig. 17), however the experimental errors are sizable.

- Consistency between $\Lambda / p$ and $k / \pi$. The same suppression factor should describe both the meson and the baryon sector. However, given our limited knowledge of mechanisms of baryon production, this test relies strongly on phenomenological models; we will return later to this subject.

Let me try to summarize the results mentioned so far:

- At the level of yseudoscalar mesons and vector mesons as primary particles, production of strange quarks in hadronization processes is suppressed compared to u quarks by a factor 0.2-0.3.

- The prccise value of $\lambda$ is quite model dependent; a perfect determination of $\lambda$ requires knowledge of the cross sections and decay modes of all resonances feeding into stable hadrons.

- Given all these problems, there is no evidence for a reaction dependence or an energy dependence of $\lambda$ (once one is well above threshold).

- The rumerical value of $\lambda$ finds a "natural" explanation in the framework of color string models. It should be obvious, however, that due to the somewhat arbitrary choice of the effective quark masses the significance of this observation is limited.

Finaliy, I want to mention some alternative approaches and one outstanding problem. As indicated before, it is not obvious that the primary objects produced in fragmentation processes are the familiar ground state mesons and baryons. Models16-19 wich primary hadronic clusters of average mass around $2-3 \mathrm{GeV}$, decaying into two mesons, another ciuster and a meson or two light clusters are capable of describing the experimental data quite well. Since a typical cluster will give rise to several mesons, the number of primary clusters in an event is significantly smaller than the equivalent 


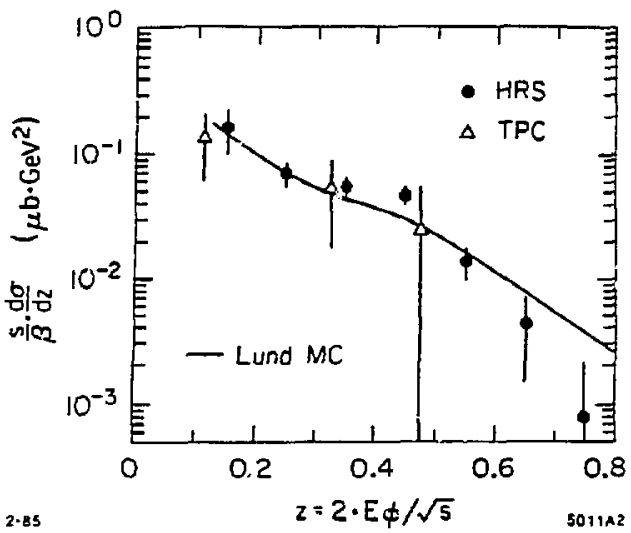

FISURE 17

Scaled cross section for $\phi$ production $e^{+} e^{-}$annihilation at $V_{s}=29$ $\mathrm{GeV}^{37}$, again compared to Lund model predictions (the model calculation uses approximately the same value of $\lambda$ as the one shown in Fig. 16).

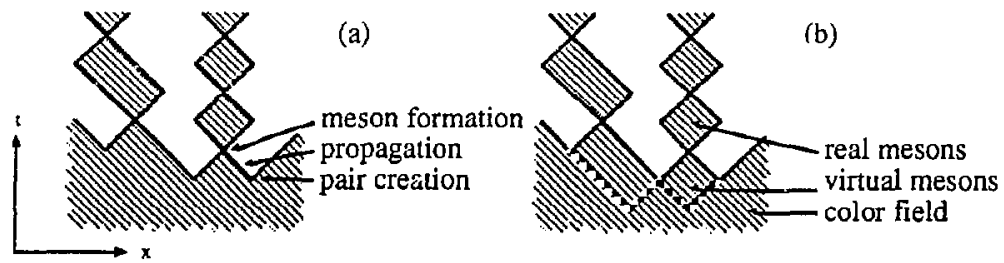

FIGURE 18

Space-time diagrams describing hadron production in string models: conventional formulation based on the string $\rightarrow$ quarks, quarks $\rightarrow$ mesons transition in a one-dimensional system of massless quarks (a) and alternative description as a direct transition strings $\rightarrow$ mesons (b). 
number of primary mesons e.g. in the Lund model. On the other hand, for cluster masses in the few-GeV range, strange-particle production is still significantly suppressed by phase space, and the number of final strange hadrons is still close to the number of strange clusters. As a consequence, less strangeness suppression is needed at the cluster level; models typically use $\lambda \approx 0.6-0.8$ in order to describe the data.

One may also question another assumption built into the models with a Fixed strangeness suppression parameter $\lambda$ : in $\equiv$ quantum mechanical system such as a color string, it is hard to see why the particle production process can be factored into two stages, namely quark pair production in the color field and successive recombination. Both processes occur on similar time scales and interference effects should be significant. After all, the newly proauced quarks "fall" immediately into a bound meson state. These arguments, which are used to justify e.g. the suppression of spin-1 mesons5, are represented in Eig. 18. The figure shows the usual space-time diagram describing quark pair production in the string. propagation of quarks until they meet a partner, and theis propagation in a "yoyo" mode as a bound meson (Fig. 18(a)). Equally well, one can however view the stage of propagation of the quark as part of tine first oscillation of the meson "yoyo" (Fig. 18(b)); Erom this point of view it appears that the color fluz tube as a whole undergoes a transition into meson states, without an intermediate quark stage. Uf course, due to the uncertainty relation the two views cannot really be distinguished, but the question remains as to which (if any) is the more appropriate classical description. In the normal string models, the inclusive cross section for a hadron $H_{i}$ is:

$\left(\frac{d \sigma}{d y d p_{T}^{2}}\right)_{\text {central region }} \sim g\left(p_{T}{ }^{2}\right) P\left(q_{i}\right) P\left(\bar{q}_{j}\right)\left|\left\langle H_{i} \mid q_{i} \bar{q}_{j}\right\rangle\right|^{2} \quad$ is

Tre Pr distribution $g$, tho quark production probabilities $P$ and the overlap integral between $q \bar{q}$ and hadron wavefunction contain adhoc parameters such as $\hat{h}$. In the spirit of Fig. $18(\mathrm{~b})$, one might 
speculate that hadron production rates might depend only on the energy (to be precise, on the transverse energy $\mathrm{E}_{\mathrm{T}}=\sqrt{\mathrm{m}^{2}+\mathrm{p}_{\mathrm{T}}^{2}}$, required to create the hadron (i.e., the length of string used up in the process), and on SU(3) Clebsch-Gordan coefficients. Stringmodel arguments suggest an $\exp \left(-b_{\mathrm{T}_{\mathrm{T}}}{ }^{2}\right.$ ) dependence (the actual expression is slightly more complicated). One and the same constant accounts for the suppression of large transverse momenta, of vector mesons, of strange mesons and Of baryons. Such a modification of the Lund string model has been studied in Ref. 40 . More or less by construction, the model retains inclusive distributions predicted by the Lund model. Amazingly enough, it predicts hadron lates for non-strange and strange mesons and baryons correctly within about 30-40\%, over a range of more than 3 orders in magnitude between frequent $(\pi)$ and very rare $(\Omega)$ particles ( $E$ ig. 19). Transverse-momentum spectra are also reproduced well. While there are certainly open questions as to the details of the implementation of this model, it demonstrates that present data cannot be used to prove conclusively that the suppression of strange hadrons actually occurs at the quark level. Much more precise measurements of production rates are needed for a better distinction!

The open problem concerns the production rate of strange mesons from scattered valence quarks in proton-proton interactions. A SFM experiment 41 measured the $\mathrm{K} / \pi$ ratio at large-p $\mathrm{p}_{\mathrm{T}}$ in a kinematic regime where the source of hadrons is almost certainly the largeangle scattering of a valence quark. In this case, the $k / \pi$ ratio is again directly related to $\lambda$. However, the experiment finds a $k / \pi$ value of $C .50$ (Fig. 20), in agreement with earlier experiments at lower energies. The large $K / \pi$ ratio can reginires a $\lambda$ of $0.55 \pm 0.05$, in disagreement with the results discussed above. We can only speculate about a source of this disagreement. Since in the ISR data ISR pions and kaons carry a large fraction (about 808) of the quarks momentum (due to the "trigger bias"), one could assume a $z$ dependence of $\lambda 42$. Since the production of large-z particles involves sizable momentum transfers, it is not unnatural to assume that the importance of mass effects is diminished. However, the neutrino experiment 27 discussed above finds no such dependence, and 


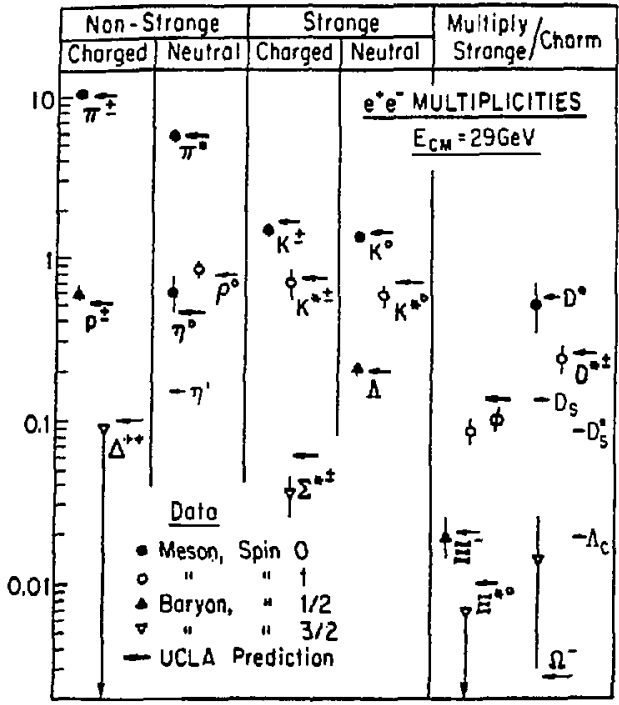

EIGURE 19

Comparison of experimental multiplicities with the predictions of the model of ref. 40 for various hadron flavors created in $\mathrm{e}^{+} \mathrm{e}^{-}$annihilation at $\sqrt{s}=29 \mathrm{GeV}$. Anti-particles are included in all cases.

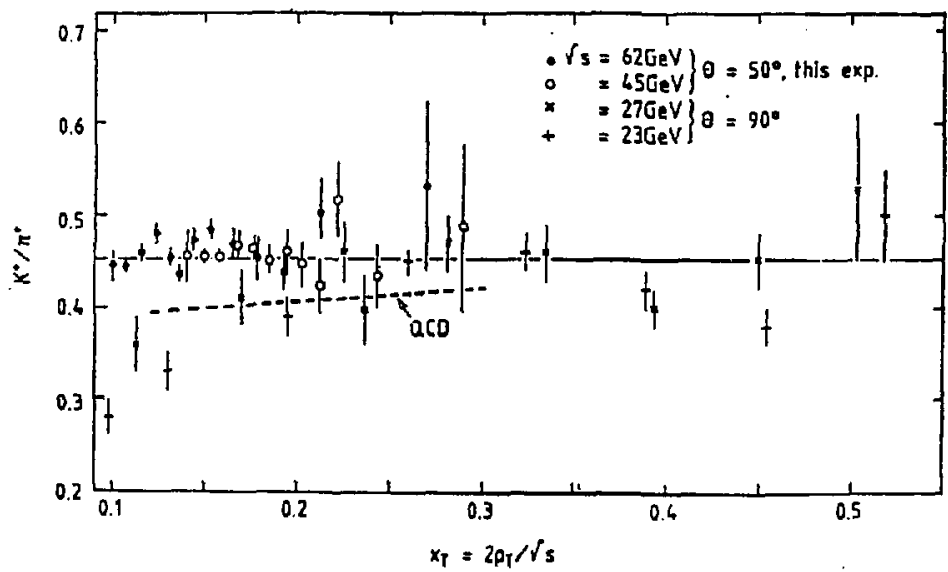

FIGURE 20

Experimental ratio of $\mathrm{K}^{+}$to $\pi^{+}$production in proton-proton interactions at large $x_{T}=2 p_{T} / \sqrt{S}^{4 l}$. The dashed line shows a model prediction using $\lambda=0.5$ 
the neutrino data and the ISR experiment appear to be inconsisttinc (Fig. 21). One remote possibility is that $\lambda$ does indeed increase with $z$, but that at the relatively low $w$ of the vp data this rise is offset by phase space effects. Another explanation is that there is another source of kaons in the ISR high pT data besides quark fragmentation. For example, it was recently pointed out 43 that higher twist contributions (i.e. direct meson production) play a significant role for some particle species (although at least for the calculation of ref. 43 and the kinematic region considered there, the increase in the kaon yield due to higher twists is relatively small). Precise measurements of particle fractions in $e^{+} e^{-}$ annihilation events at $29 \mathrm{GeV}$ cms energy will soon be available 44 and may help to solve this puzzle.

3. STRANGENESS PRODUCTION MECHANISMS AND CORRELATIONS BETWEEN STRANGE PARIICLES.

In the investigation of parton fragmentation, the study of particle correlations can give information beyond that obtainable from inclusive spectra. As discussed in the introduction, the strangeness quantum number can be used to label a specifiz quark pair and tc study the effect of confincment forces on those quarks. Typically, correlations are studied in the following way: one selects events with a "test particle", say a $\mathrm{K}^{-}$, in a small, fixed rapidity interval, and then in those events considers the number of additional kaons produced as a function of their rapidity, or as a function of the rapidity difference to the "test" kaon. Fig. 22 shows KK correlation functions in $e^{+} e^{-}$annihilation events 45 and in hadronic interactions ${ }^{46}$. In each case, the correlation function is defined as the measured number of additional kaons per event divided by the number of kaons found in the same kinematic interval in average events without the "test kaon" requirement:

$$
c=\left(\frac{1}{\sigma} \frac{d^{2} \sigma}{d y_{K} d y_{\text {test }}}\right) /\left(\frac{1}{\sigma^{2}} \frac{d \sigma}{d y_{K}} \frac{d \sigma}{d y_{\text {test }}}\right)-1
$$

This definition yields $\mathrm{C}=0$ for uncorrelated production. We observe a strong positive correlation of opposite-sjgn kaons at small rapidity differences. The positive correlation is simply a reflection 


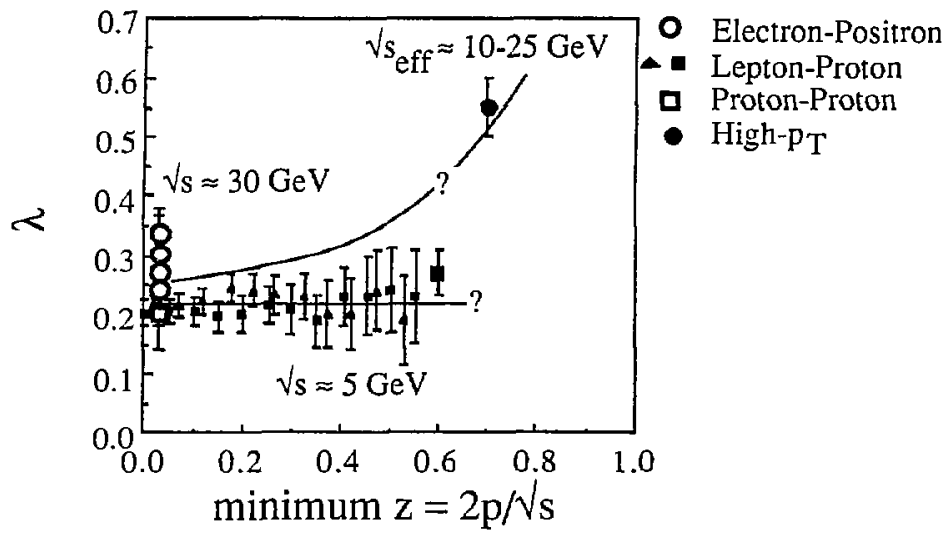

FIGURE 21

Values of $\lambda$ as derived from $k / \pi$ ratios, as a function of the minimum $z=$ Phadron/pquark above which hadrons are used in the analysis. Data are shown for $e^{+} e^{-}$annihilation (from several experiments), for neutrino-nucleon scattering, for low-pT pp reactions and for high-pT meson production in PP interactions.
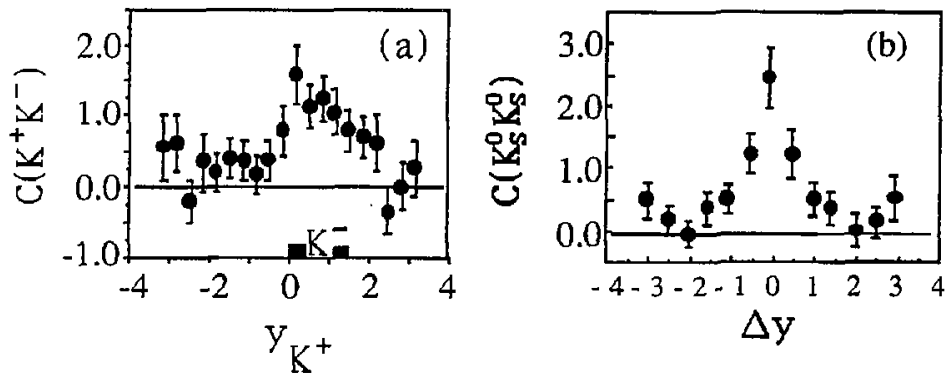

FIGURE 22

(a) Correlation function $C$ for unlike-sign kaon production in $e^{+} e^{-}$ annihilation events at $V_{s}=29 \mathrm{GeV} 45$, as a function of the rapidity of one kaon, while the other one is fixed in the interval $0<y<1.5$. (b) Correlation between neutral kaons produced in hadronic interactions, as a function of the rapidity difference 46 . 
of strangeness conservation; however the fact that the excess of opposite-sign kaons is localized at the same rapidity as the test particle indicates that confinement does indeed involve only small momentum transfers and does not separate quark pairs over large distances in phase space (i.e. rapidity).

An interesting result is obtained if the test particle is moved towards high rapidity into the fragmentation region of $e^{+} e^{-}$events (Fig. 23). Now there are indications of two peaks in tire correlation function, one local peak and one peak at large and opposite rapidity, indicating that two different mechanisms contribute to the production of strange-quark pairs: one low-q $\mathrm{q}^{2}$ mechanism and one mechanism creating large rapidity separations, i.e. large momentum transfers. Of course, the latter mechanism is the initial hard process creating the primary quark-antiquark pair from the virtual

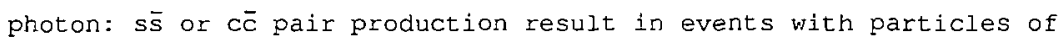
opposite strangeness in the two fragmentation regions. The low- $\mathrm{q}^{2}$ process is then the color confinement; in this case the strange quarks are produced from the breakup of the color field.

These kaon correlations are consistent with expectations based on the string model; however they do not prove conclusively that hadronization does indeed proceed along a quasi-onedimensional string. A correlation effect more characteristic for string models was observed recently 47 in a study on baryon correlations: in string models, the rapidity $y$ of a particle is closely related to the position of the production points of its partons along the string. It is hence difficult to create particles with identical quantum numbers (such as charge, strangeness, or baryon number) at the same rapidity. Both along the string and in $y$ the signs of quantum numbers tend to alternate; a strange particle is followed by an anti-strange particle, a baryon by an antibaryon etc. string models therefore predict an anticorrelation between two like-sign pions, kaons, or protons. Unfortunately, this anticorrelation is virtually unobservable for pions, since most $\pi$ 's result from resonance decays, and (anti-) correlations are washed out, and since Bose-Einstein correlations further enhance production of pion pairs with small momentum differences. The anticorrelation is observed 
for baryons 47 , where resonance smearing plays a negligible role (Fig. 2A(a)). A similar (however somewhat weaker) anticorrelation shows up for like-sign kaons produced in 2-jet events (Eig. 24 (b)). For this analysis, 3 -jet events with radiative gluons have been explicitly excluded, since an additional large-angle jet gives rise to very strong correlations, completely wiping out the weak anticorrelation. The like-sign "repulsion" is not as pronounced for kaons as as it is for protons (where it is visible without aditional cuts); indeed string models predict the effect to increase with rising hadron mass 47 .

In summary, string models provide a surprisingly good phenomenology of not only of inclusive strange-particle production, but also of correlations between strange particles.

\section{BARYON PRODUCTION}

As cutlined earlier, baryons offer additional degrees of freedom, which are valuable in a systematic study of confinement processes. At this point, the only models of baryon production which have survived the experimental tests are the diquark model and its variants $4 \mathrm{~d}^{-52}$. These models assume that occasionally a diquark-antidiquark pair is produced in the confinement process. Since a dicuark can act as an effective color antitriplet, it may combine with a quark to form a baryon. So far, it is not completely clear if such diquarks represent tightly-bound objects or if two looselyinteracting guarks merely act as some kind of "effective diquark". Diquark models predict 48,49 a strong suppression of baryons with spin-l diquarks, or with strange diquarks, since diquark creation rates in a color field depend exponentially on the square of the diquark mass - see Egn. 4. A strange diquark is hence more strongly suppressed (compared to a non-strange diquark) than a strange quark. (compared to a non-strange quark). While the notion of pointlike diquarks may seem far fetched, detailed mechanisms have been described ${ }^{3}$ of how effective diquarks can form. Baryon production is best scudied in processes without incident baryons, such as $e^{+} e^{-}$ annihilation. While many papers on proton and $\Lambda$ production in highenergy $\mathrm{e}^{+} \mathrm{e}^{-}$interactions at $P E P$ and PETRA energies nave been pub- 

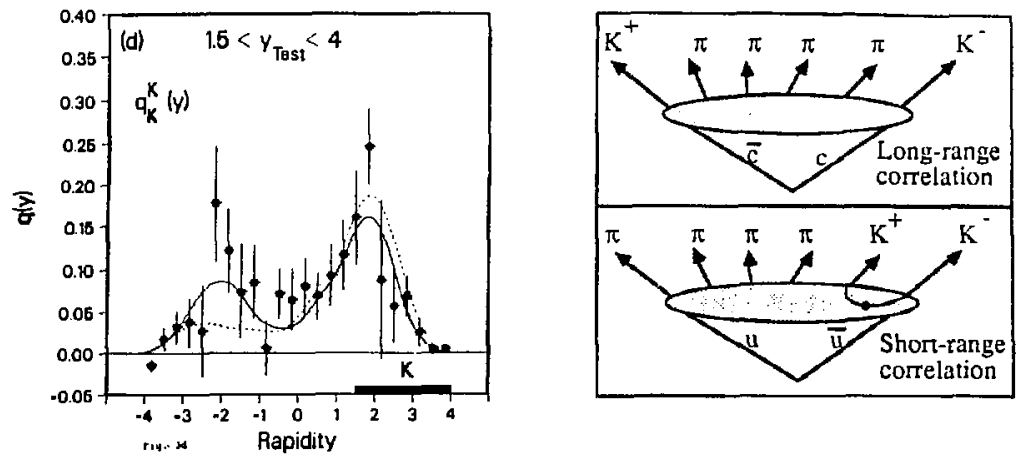

FIGURE 23

Net strangeness per unit rapidity seen in charged kaons, for $e^{+} e^{-}$ annihilation events with a selected $k^{-}$at large rapidity $y>1.5$ 45. The quantity shown measures where in rapidity the strangeness of the $K^{-}$is compensated and is roughly equivalent to the difference between the correlation functions for opposite-sign and like-sign kaon pairs. The curves show predictions of different fragmentation models. The diagram to the right indicates the sources of long-range and short-range kaon correlations.

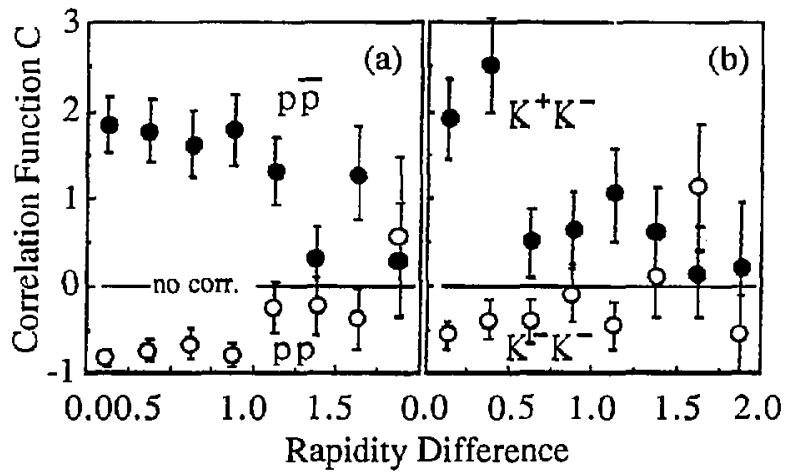

FIGURE 24

(a) Correlation function $C$ for proton-antiproton pairs and for proton-proton pairs in $e^{+} e^{-}$annihilation events at $V_{s}=29 \mathrm{GeV} 47$, as a function of the rapidity difference between the two baryons. (b) same for unlike-sign and like-sign kaon pairs in 2-jet events. 
lished 14,53-55, the most concise data set on strange baryon production comes from the ARGUS experiment 56 at the DORIS storage ring. In order to catract strangeness suppression etc., I fitted the ARGuS data using a diquark model with Su(6) symetry broken by suppression factors for spin-1 diquarks and for strange diquarks 58 . For $\lambda$-values in the range discussed before, $\lambda=0.2-0.3$, this model works quite well and yields a strange-diquark suppression considerably bigger than $\lambda: \mathrm{P}\left(\mathrm{us}_{0}\right) / \mathrm{P}\left(\mathrm{ud}_{0}\right)=0.10 \pm 0.04$ (the index 0 indicates the diquark spin). As expected, all spin-1 diquarks are Fomi to be suppressed by at least a factor 50 compared to the lignosist diquark, a spin-0 ud system.

\section{FOLAETZATION}

Both string models and QCD calculations predict significant polarization effects in the hadronization process; basically, baryons sholid be polarized perpendicular to the transverse momentum of the racion and to the direction of the color field49. Such polarization is incoed soserved for leading baryons in hadronic collisions, and is contont $y=h$ the predictions 39 . A quite complete discussion cf vist: ation effects in hadronic reactions can be found elsewhen in these proceedings; I will not repeat the discussion here. [etection of baryon polarization in $e^{+} e^{-}$annihilation, where the configuration of the color field is (theoretically) much better kncwn would provide a further excellent test of string models. Unforcunately, measurement of the polarization requires knowledge of the color field direction, i.e. knowledge as to which jet is the quark jet and which the antiquark jet. Algorithms to identify these jets have been developed, but so far only upper limits on $\Lambda$ polarization have been given52. It is at this point not clear whether these limits are in contradiction with model predictions or not.

6. SUMAR:

In sumiary, one can state that the production of strange hadrons in ingh-energy reactions provides an extremely valuable and not yet fully exploited tool to study the dynamics of flavor production in confinement processes. So far, the one-dimensional color string 
provides a rather convincing phenomenology of hadron production in general, and of strange particle production in particular. Although in typical implementations of the string model strangeness suppression factors are treated as free parameters, the resulting values of the parameters can be explained in terms of quark mass effects. However, more and better measurements are needed to rigorously test model predictions beyond the present 10-20\% level of accuracy.

\section{DISCLA IMER}

This paper is supposed to serve as an introduction to the topic, it is not exhaustive as far as the discussion of data and models or the list of references is concerned. Emphasis is placed on strangeness production in quark fragmentation and its description in the very popular framework of string models; because of length limitations, many interesting related topics, such as the discussion of beam and target fragmentation in hadronic reactions, or of alternative models for hadron production are omitter or abbreviated.

\section{ACKNOWLEDGEMENTS}

This work was supported by the U.S. Department of Energy under Contract No. DE-AC03-76SE00098 and by the National Science Eoundation. The author acknowledges receipt of an A.P. Sloan Fellowship.

\section{REFERENCES}

1) J. Schwinger: Phys. Rev. 82, 554

2) A. Casher, H. Neuberger, S. Nussinov: Rhys. Rev. D20 (1979) 179

3) A. Casher, H. Neuberger, S. Nussinov: Phys. Rev. D21 (1980) 1966

4) H. Neuberger: Phys. Rev. D20 (1979) 2936

5) B. Andersson et al.: Phys. Rep. 97 (1983) 31

6) E. Brezin, C. Itzykson: Phys. Rev. D2, 191 (1970)

7) E.G. Gurvich: Phys. Lett. 87B (1979) 386

8) G. Schitrnolz, M. Teper: Z. Phys. Cl3 (1982) 53

9) K.W. Bell et al.: RL-820-011 (unpublished) 
10) B. Andersson, G. Gustafson, G. Ingelman: Phys. Lett. 855 (1979) 417

11) J.D. Bjorken: Int. Conf. on Physics in Collision, Stockholm, Sweden, 1982; and EERMILA3-COnf-82/42-THY

12) TAssc Culab., M. Althofi et al,: 2. Phys. C22 (1984) 307

13) D. 2xifued al., nucl. prys. B155 (1979) 269

14) Tasso collab., M. Althoff et $\equiv 1$. : Z. phys. C27 (1985) 27

15) V.V. Antsovich, M.E. Koorinsky: Phys. Lete. 523 (1974) 217

16) B.R. Hebber: Muci. Phys. 3238 (1984) 492

17) T.D. Gottschalk, Nucl. Phys. B214 (1983) 201; B239 (1984) 325; $\therefore 239(1984) \quad 349$

18) G.C. EON, S. wolfram: Nucl. Phys. Bl68 (1980) 285

19) R.D. Field, S, Wolfram: Nucl. Phys. B213 (1983) 65

20) R. Hageciorn, Proc. Erice 1972, p. 255

21) A. Wrodlewski, Acta Phys. Pol. 316 (1985) 379

22) We used JETSET Vs. 5.3, see T. Sjöstrand, M. Bengtsson: LU-TP $36-22(1996)$

23) JADE Co:lád., W. Bartel et al.:Z. Phys. C20 (1983) 187

24) Yark II Collab. H. Schellman et al: SLAC-PUB 3448 (1984)

25) HRS Collab.: Phys. Lett. $158 \mathrm{~B}$ (1985) 519

26) TPC Ccllab.: H. Aihara et al., to be published

27) G.T. Jores et al.: Z. Phys. C27, 43 (1985)

28) V. Ammosov et al.; Phys, Lett. 938 (1980) 210

29) EMC Collaboration, M. Arneodo et al., Z. Phys. C34 (i987) 283

30) N.J. Baker et al.: Phys. Rev. D34, 1251 (1986)

31) EMC Collaboration, M. Arneodo et al., Ehys. Lett. 1458 (1984) 156

32; I. Cohen at a1.: Phys. Rev. Lett. 40 (1978) 1614

33) G.J. Alner et al.: Nucl. Phys. B258 (1985) 505

34) TPC Collab., H. Aihara et al.: Phys. Lett. B 184 (1987) 114

35) C. Petersen, LU-TP 85-15 (1985)

36) HR. Collab., M. Derrick et al.: Phys. Lett. 1585 (1985) 519

37) HRS Collab., M. Derrick et al.: Phys. Rev. Lett. 54 (1985) 2558

38) P.K. Malhotra, R. Orava: Z. Phys. Cl7 (1983) 85.

39) K.J. Heller, Proc. High Energy Spin ehysics, Marseille (1984) 
40) C.D. Buchanan, S.E. Chun: UCLA 87-005, subm, to Phys. Rev. Let.

41) A. Breakstone et al.: Z. Phys. C23 (1984) 9

42) N. Geist, CERN-EP/87-127, subm. to Phys. Lett. B

43) G. Ingelman: DESY 86-131 (1986)

44) TPC Collab,, H. Aihara et a1.: in preparation

45) TPC Collao., H. Aihara et al.: Phys. Rev. Lett. 53 (1984) 2199

46) R. Harris et al.: Rhys. Rev. D18 (1978) 92

47) TPC Collab., H. Aihara et aI.: Phys. Rev. Lett. 57 (1986) 3140

48) B. Anderssor, G. Gustafson, T. Sjöstrand: Nucl. Fhys. B197 (1932) 45

49) B. Andersson, G. Gustafson, T. Sjöstrand: Physica Scripta 32 (1985) 574

50) T. Meyer, 2. Phys. C12 (1982) 77

51) E.M. Ilgenfritz, J. Kripfganz, A. Schiller: Acta Phys. Pol. B9 (1978) 881

52) A. Bart1, H. Eraas, W. Majoretto: Phys. Rev. D26 (1982) 1061

53) IPC Coulab., H. Aihara et al.: Phys. Rev. Lett. 54 (1985) 274

54) E. Barfinger et al.: Fhys. Rev. Lett. 56 (1986) 1346

55) D.H. Säron, Proc. Int. Conf, on High Energy Physics, Bari (1985)

55) AnGUS Collab., H. 'brecht et al.: Phys. Lett. 183B (1987) 419

57) Cleo Collab., S. bu rends et al.: Phys. Rev. D31 (1985) 2161

58) TAsso Collad., M. Althoff et al.: Z. Phys. C26 (1984) 181 